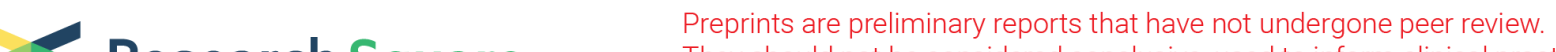 $\begin{array}{ll}\text { Research Square } & \text { They should not be considered conclusive, used to inform clinical practice, } \\ \text { or referenced by the media as validated information. }\end{array}$
}

\section{DNA Aptamers Specific for Legionella Pneumophila: Systematic Evolution of Ligands by Exponential Enrichment in Whole Bacterial Cells}

\section{Lina Xiong}

Sun Yat-sen University

\section{Mingchen Xia}

Guangzhou Saite Testing Co., LTD

\section{Qinglin Wang}

Sun Yat-Sen University

\section{Zhen Meng}

Zhongkai University of Agriculture and Engineering

\section{Jie Zhang}

Zhongkai University of Agriculture and Engineering

\section{Guohui Yu}

Zhongkai University of Agriculture and Engineering

\section{Zhangyong Dong}

Zhongkai University of Agriculture and Engineering

\section{Yongjun Lu}

Sun Yat-Sen University

Yunhao Sun ( $\nabla$ sunyunhaoscope@163.com )

Zhongkai University of Agriculture and Engineering https://orcid.org/0000-0003-4895-771X

\section{Research Article}

Keywords: Legionella pneumophila, Aptamer, SELEX, Pathogen detection

Posted Date: January 7th, 2022

DOI: https://doi.org/10.21203/rs.3.rs-1167280/v1

License: (c) (i) This work is licensed under a Creative Commons Attribution 4.0 International License. Read Full License

Version of Record: A version of this preprint was published at Biotechnology Letters on April 13th, 2022. See the published version at https://doi.org/10.1007/s10529-022-03252-z. 


\section{Abstract}

Legionella pneumophila is the major causative agent of Legionnaires' disease and Pontiac fever, which pose major public health problems. Rapid detection of L. pneumophila is important for global control of these diseases. Aptamers, short oligonucleotides that bind to targets with high affinity and specificity, have great potential for use in pathogenic bacterium detection, diagnostics, and therapy. Here, we used a whole-cell SELEX (systematic evolution of ligands by exponential enrichment) method to isolate and characterize single-stranded DNA (ssDNA) aptamers against $L$. pneumophila. A total of 60 ssDNA sequences were identified after 17 rounds of selection. Other bacterial species (Escherichia coli, Bacillus subtilis, Pseudomonas syringae, Staphylococcus aureus, Legionella quateirensis, and Legionella adelaidensis) were used for counterselection to enhance the specificity of ssDNA aptamers against $L$. pneumophila. Four ssDNA aptamers showed strong affinity and high selectivity for $L$. pneumophila, with $K_{\mathrm{d}}$ values in the nanomolar range. Bioinformatic analysis of the most specific aptamers revealed predicted conserved secondary structures that might bind to L. pneumophila cell walls. In addition, the binding of these four fluorescently labeled aptamers to the surface of L. pneumophila was observed directly by fluorescence microscopy. This is the first study to use SELEX to target $L$. pneumophila whole cells. The aptamers identified in this study could be used in the future to develop medical diagnostic tools and public environmental detection assays for L. pneumophila.

\section{Introduction}

Legionella species, the major causative agents of Legionnaires' disease and Pontiac fever, are commonly found in environmental samples (Xiong et al., 2016). They have been shown to induce lung infection and cause dysfunction in other organs, such as the heart and kidney, and in the central nervous system (Xiong et al., 2015). Legionella pneumophila specifically has been identified as the primary cause of Legionnaires' disease. Early detection of L. pneumophila would allow for faster recovery and a reduction in the number of fatal cases. Additionally, monitoring the L. pneumophila population in the environment could reduce the likelihood of Legionnaires' disease outbreaks (Xiong et al., 2017). However, traditional culturing methods used for detecting L. pneumophila in samples rely on enrichment and selective plating followed by biochemical, serological, and molecular identification. These methods take 2 to 3 days to obtain a result and are labor intensive and tedious (Rajendhran and Gunasekaran, 2011). Recently, highly specific detection techniques, such as ELISA, PCR, and real-time qPCR, have shortened detection time. However, these methods depend on complex and technically difficult steps and are prohibitively expensive for routine testing (Woo et al., 2008). Biosensors have demonstrated a potential for rapid bacterial detection but rely on nanomaterials or complex platforms such as functional magnetic nanoparticles, electrochemical analysis systems, and field-effect transistors (Gopinath et al., 2014). Antibodies have also been applied for more than 40 years in assays for microbial detection but have many limitations despite their widespread application. For instance, the synthesis of antibodies requires a host animal, antibodies may be unstable at room temperature, and antibodies will not be obtained when target chemicals are toxic to the host organism. 
Aptamers are single-stranded DNAs (ssDNAs) or RNAs that can bind to a wide range of non-nucleic acid targets with high affinity and specificity (Keefe et al., 2010). In detection and diagnostic assays, aptamers have several advantages over antibodies (Keefe et al., 2010). For instance, aptamers are obtained through an in vitro selection process, which is achieved at a lower cost and with less batch variation than in vivo antibody production. Due to their high stability at a range of temperatures and pHs, aptamers are also relatively easy to handle. In addition, aptamers can be easily modified with dyes or other functional groups to be labeled or immobilized on a substrate (Syed and Jamil, 2018).

Aptamer sequences are usually identified through the SELEX (systematic evolution of ligands by exponential enrichment) process, which starts with incubating the ssDNA or RNA library with the target, followed by separation and exponential amplification of the binding ssDNA or RNA (Torres-Chavolla and Alocilja, 2009). By repeating this process 8-20 times before cloning and sequencing the oligonucleotides that bind to the target, the most suitable aptamers can be identified (Dua et al., 2016). Aptamers can be selected for a wide variety of targets, from small molecules to whole cells (Bruno, 2015; Cao et al., 2009; Sypabekova et al., 2017). In recent years, aptamers have been selected against cells from different bacterial species, including Escherichia coli K88 (Peng et al., 2014), Salmonella typhimurium (Duan, Wu, et al., 2013; Dwivedi et al., 2013; Moon et al., 2013), S. enteritidis (Kolovskaya et al., 2013; Park et al., 2014), S. paratyphi A (Yang et al., 2013), Vibrio parahaemolyticus (Duan et al., 2012; Tang et al., 2013), Listeria monocytogenes (Suh et al., 2014; Suh and Jaykus, 2013), Shigella dysenteriae (Duan, Ding, et al., 2013), Staphylococcus aureus (Cao et al., 2009), Proteus mirabilis (Savory et al., 2013), Pseudomonas aeruginosa (Wang et al., 2011), Mycobacterium tuberculosis (Chen et al., 2007), and Campylobacter jejuni (Dwivedi et al., 2010). However, no specific aptamers have been reported for L. pneumophila.

In this study, we isolated four sequences for DNA aptamers specific to L. pneumophila using a whole bacterial cell SELEX process. The predicted secondary structures of selected aptamers were analyzed, and the binding of these aptamers to target cells was observed by fluorescence microscopy. In addition, we characterized their affinity and selectivity for $L$. pneumophila cells. The results provide an important reference for pathogen detection using aptamers in the future.

\section{Results}

\section{In vitro selection of aptamers}

To select aptamers that bind to Legionella pneumophila, we applied a cell-SELEX strategy using a library of ssDNA sequences containing a 45-base random region flanked by two defined primer binding regions (5'-GCAATGGTACGGTACTTCC-N45-CAAAAGTGCACGCTACTTTGCTAA-3'; Table 1). A total of 17 rounds of selection were performed until the ssDNAs binding to the target cells dominated the DNA pool (Fig. 1).

The genus Legionella includes more than 57 species (including subspecies) and more than 70 serogroups, not all of which are associated with human disease. The species most frequently detected in diagnosed cases is L. pneumophila, consisting of 16 serogroups (Casini et al., 2017); L. pneumophila serogroup 1, responsible for the first identified outbreak in 1976 in Philadelphia (USA), is the cause of 
95\% of infections in Europe and $85 \%$ of infections worldwide (Cloutman-Green et al., 2019). The phylogenetic diversity of this Philadelphia-1 strain has since expanded, now including the JR32 and Lp02 strains (Schmolders et al., 2017) commonly used for L. pneumophila research (Maita et al., 2016). In this study, JR32 and Lp02 were used as the target strains for aptamer selection. Live cells were used because the conformation of cell wall molecules, which represent the most likely aptamer targets, may change when cells die. Also, considering bacterial cell wall composition changes during growth in culture, we used stationary-phase bacterial cells in all experiments. Following incubation, the target-bound aptamers were eluted and enriched at each selection round by amplification using PCR. After each round of selection, the obtained ssDNAs were quantified by a spectrophotometer and analyzed by agarose gel electrophoresis (Fig. 1A).

With each SELEX round, the percentage of SSDNA binding to the target cells gradually increased. However, there were slight drops in elution yield after the second and sixth rounds, most likely due to counterselection (Fig. 1B). During SELEX, four counterselection processes were performed to increase the selectivity of aptamers to the target cells using a mixture of other bacterial species (Escherichia coli, Bacillus subtilis, Pseudomonas syringae, Staphylococcus aureus, Legionella quateirensis, and Legionella adelaidensis) at the second, sixth, ninth, and thirteenth rounds of selection. After the 17 rounds of selection, ssDNA bound to target cells dominated the DNA pool with about $90 \%$ elution yield. A total of 60 cloned transformants ( $E$. coli cells harboring a vector that contained the aptamer sequences) were sequenced to identify specific aptamer candidates. From these transformants, we identified four unique aptamer sequences (S11, S25, S28, and S29) that were specific to LP02 cells and JR32 cells (Table 2).

\section{Sequencing and bioinformatics analysis}

The sequences of the S11, S25, S28, and S29 L. pneumophila aptamers are listed in Table 2. The computed GC/AT ratio of all four aptamers varies from 1.63 to 0.96 . Since the GC/AT ratio is one of the most basic sequence characteristics in terms of nucleotide composition, aptamer sequences can be modified and optimized in future studies by taking this ratio into account. To understand the impact of aptamer sequence structure on binding, we generated predicted secondary structures for the most efficient L. pneumophila-binding aptamers using the mfold algorithm. Aptamers S11 and S29 had similar predicted stem-loop region secondary structures, which contained GGGCA residues at the apical loops (Fig. 2). S25 and S29 also contained a nearly identical sequence, CAxCTGTA. In addition, S11, S25, and S28 all shared one conserved stem loop with TACTT residues, while S28 and S29 had an identical stem loop containing the sequence CAAAAGTG (Fig. 2).

For the aptamers S11, S25 and S28, mfold yielded four predicted secondary structures, while five different alternative conformations were predicted for S29. However, the conserved loops in different aptamers were stable in different predicted secondary structures, which suggests that these features could play an important role in the interaction of aptamers with their ligands in the bacterial cell wall.

\section{Assessment of selectivity}


To determine that the selected aptamers were capable of binding $L$. pneumophila cells, the four $L$. pneumophila-binding DNA aptamers (S11, S25, S28, and S29) were examined using the same concentration of aptamers ( $250 \mathrm{nM})$ and visualized by fluorescence microscopy. Other bacterial species (E. coli, B. subtilis, P. syringae, S. aureus, L. quateirensis, and L. adelaidensis) were used as negative controls. As shown in Fig. 3A, fluorescently labeled L. pneumophila JR32 and Lp02 cells were observed with 6-carboxyfluorescein (FAM)-labeled S29, while the negative control cells incubated with FAM-S29 did not show any definite fluorescence signal on cells. Using Multiscan Spectrum, the intensity of fluorescence was detected. All four of the aptamers showed selective binding to L. pneumophila JR32 and $L p 02$, and they had very low binding capacity for other bacterial species, including $L$. quateirensis and $L$. adelaidensis cells (Fig. 3B). These results indicate that the four SsDNA aptamers (S11, S25, S28, and S29) showed affinity and high selectivity to L. pneumophila JR32 and Lp02.

\section{Determination of equilibrium dissociation constants}

To evaluate aptamer binding in more detail, we determined the affinity of individual aptamers (S11, S25, S28, and S29) to L. pneumophila JR32 and Lp02. The fluorescein-labeled ssDNA aptamers (in increasing concentrations of $12.5,31.25,62.5,125,250$, and $500 \mathrm{nM}$ ) were incubated with $10^{7}$ JR32 or Lp02 cells for $60 \mathrm{~min}$ at $20^{\circ} \mathrm{C}$ with mild shaking. The fluorescence intensity of each sample was measured with a fluorospectrophotometer, and $K_{\mathrm{d}}$ values were determined by nonlinear regression analysis using Origin 9.5 software. As shown in Fig. 4, computed $K_{\mathrm{d}}$ values of all four aptamers for L. pneumophila JR32 and Lp02 varied from 12.98 to $35.00 \mathrm{nM}$. Aptamer S29 had the highest affinity for L. pneumophila JR32 cells, with a low $K_{\mathrm{d}}$ value $(12.98 \mathrm{nM})$, while $\mathrm{S} 28$ had the lowest $K_{\mathrm{d}}$ value $(22.11 \mathrm{nM})$ for L. pneumophila Lp02 cells.

\section{Discussion}

L. pneumophila is an opportunistic pathogen comprising more than 16 serotypes, with serogroup 1 being responsible for most serious infections such as Legionnaires' disease, causing $95 \%$ of infections in Europe and 85\% of infections worldwide (Casini et al., 2017; Cloutman-Green et al., 2019). In our study, the representative strains JR32 and Lp02 (Maita et al., 2016) were used as target cells to develop specific, high-affinity aptamers for pathogenic L. pneumophila. Notably, the binding affinity of each aptamer varied between these two strains (Fig. 3 and 4). These results may be due to experimental error during assessments of affinity and specificity for aptamers against JR32 and Lp02 cells. However, it is also possible that, although JR32 and Lp02 were isolated simultaneously, the two strains currently display distinct genomic features leading to differences in cell wall composition (Maita et al., 2016).

In this study, we determined that using fluorescein-modified primers to amplify the aptamers makes the product more visible under ultraviolet light, which aids in product recovery. In addition, to avoid primer contamination during the SELEX process we used a gel extraction kit to purify and recover products. The fluorescein group may help increase the recovery efficiency during purification and recovery steps. 
Using the mfold algorithm, we generated predicted secondary structures for the most efficient $L$. pneumophila-binding aptamers (Fig. 2). We observed that in the constant region primer, S11, S25, and S28 share one conserved stem loop with TACTT residues. In-depth analysis revealed that the generations of these conserved secondary structures were due to site mutations in the constant primer regions of these aptamers during the PCR amplification process (Fig. 2). These results demonstrated that the conserved stem loop with TACTT residues could be the key secondary structures that allow these aptamers to bind the target cells. In future studies, we will test this hypothesis by truncation or sitedirected mutation assays. In addition, elucidation of the specific binding targets on the cells themselves may also help us better understand this mechanism.

To our knowledge, this is the first study to report the binding of aptamers to L. pneumophila. Importantly, some of the aptamers described here recognize pathogenic L. pneumophila strains with affinity and specificity suitable for potential use in clinical diagnosis, therapeutics, and environmental pathogen detection applications.

\section{Conclusions}

We applied the whole-cell SELEX (systematic evolution of ligands by exponential enrichment) method to isolate and characterize single-stranded DNA (ssDNA) aptamers against $L$. pneumophila. Four ssDNA aptamers were obtained and showed strong affinity and high selectivity for $L$. pneumophila, with $K_{\mathrm{d}}$ values in the nanomolar range. Bioinformatic analysis of the most specific aptamers revealed predicted conserved secondary structures that might bind to L. pneumophila cell walls. In addition, the binding of these four fluorescently labeled aptamers to the surface of $L$. pneumophila was observed directly by fluorescence microscopy. The aptamers identified in this study could be used in the future to develop medical diagnostic tools and public environmental detection assays for L. pneumophila.

\section{Materials And Methods}

\section{Microorganisms and culture conditions}

Escherichia coli (DH5a), Bacillus subtilis (13407), Pseudomonas syringae (DC3000), Legionella pneumophila (Lp02, Philadelphia-1, thyA, rpsL, hsdR), Legionella pneumophila (JR32, Philadelphia-1, JR32), Legionella quateirensis (27-1), Legionella adelaidensis (49625), and Staphylococcus aureus (26111) were obtained from the American Type Culture Collection (ATCC). Cells were cultured in Luria broth (LB) or AYET medium at $37^{\circ} \mathrm{C}$. Growing cultures $\left(\mathrm{OD}_{600}\right.$ of 3.0$)$ were used in all experiments. Cells were washed three times with wash buffer (PBS, $\mathrm{pH}=7.5,0.1 \%$ Tween 20) before use.

\section{Aptamer selection process (SELEX)}

A whole-cell SELEX approach was used to select 6-carboxyfluorescein (FAM)-labeled sSDNA aptamers with binding affinity and specificity to L. pneumophila. The method of Dwivedi et al. (2010) was used, with minor modifications. To initiate the selection process, an 88-mer combinatorial ssDNA library (5'- 
GCAATGGTACGGTACTTCC-N45-CAAAAGTGCACGCTACTTTGCTAA-3') procured from Ruibiotech (Guangzhou, PRC) was amplified using the FAM-labeled forward constant region primer (5'-FAMGCAATGGTACGGTACTTCC-3') and reverse

constant region primer (5'-TTAGCAAAGTAGCGTGCACTTTTG-3') in $2 \times$ Phanta Master Mix (Vazyme, Nanjing, PRC). Random library nucleotides (approximately $10^{14}$ molecules) diluted in selection buffer (PBS, $\mathrm{pH}=7.5,0.05 \%$ Tween 20 ) were denatured for $10 \mathrm{~min}$ at $96^{\circ} \mathrm{C}$, cooled for $10 \mathrm{~min}$ at $4^{\circ} \mathrm{C}$, and incubated with $1 \mathrm{~mL}$ of $10^{8}$ colony forming units (CFU)/mL of bacterial cells for $45 \mathrm{~min}$ at $20^{\circ} \mathrm{C}$ under constant agitation (30 rpm). The bacterial cells were concentrated by centrifugation $(6000 \mathrm{~g}, 3 \mathrm{~min})$ and washed three times with wash buffer (PBS, $\mathrm{pH}=7.5,0.1 \%$ Tween 20 ) to remove unbound and nonspecifically bound aptamers. The aptamers bound to cells were eluted by resuspending the bacteriaaptamer complex in sterile water and heating it to $95^{\circ} \mathrm{C}$ for $5 \mathrm{~min}$. The supernatant was collected and amplified by PCR in a 100- $\mu \mathrm{L}$ reaction, using $2 \times$ Phanta Master Mix (Vazyme, Nanjing, PRC) with $0.4 \mu \mathrm{M}$ FAM-labeled forward constant region primer and $0.4 \mu \mathrm{M}$ P-reverse constant region primer. Amplification conditions were as follows: $96^{\circ} \mathrm{C}$ for $2 \mathrm{~min}$ (denaturation and heat activation of polymerase); 12 cycles of a) $96^{\circ} \mathrm{C}$ for $20 \mathrm{~s}$, b) $56^{\circ} \mathrm{C}$ for $33 \mathrm{~s}$, and c) $72^{\circ} \mathrm{C}$ for $24 \mathrm{~s}$ (annealing and extension); $72^{\circ} \mathrm{C}$ for $5 \mathrm{~min}$ (final extension); and $6^{\circ} \mathrm{C}$ for 2 min (end of amplification). Products were resolved by $2 \%$ agarose gel. To obtain ssDNA, PCR products were obtained by digestion with 5 units of lambda exonuclease (New England Biolabs, Ipswich, MA, USA) per $50-\mathrm{mL}$ reaction. Reactions were incubated for $30 \mathrm{~min}$ at $37^{\circ} \mathrm{C}$ and then heat inactivated for $5 \mathrm{~min}$ at $80^{\circ} \mathrm{C}$. Digested products were extracted using the D205 StarPrep Gel Extraction Kit (Genestar, Beijing, PRC) and used for the next round of SELEX. Concentration of SSDNA was measured using a NanoDrop 1000 Spectrophotometer (ThermoScientific, DE, USA). For counter-SELEX, the candidate aptamer pool obtained after multiple rounds of SELEX was incubated with a cocktail of nontarget bacterial cells [E. coli (DH5a), B. subtilis (13407), P. syringae (DC3000), L. quateirensis (27-1), L. adelaidensis (49625), and Staphylococcus aureus (26111)] at a pooled concentration of $10^{8} \mathrm{CFU} / \mathrm{mL}$. In this case, the cell-bound aptamer molecules were discarded, and the unbound aptamer pool was recovered by centrifugal washing. After completion of all 17 rounds of SELEX and counter-SELEX processes, the final aptamer pool was enriched by PCR with forward constant region primer and reverse constant region primer. Products were cloned using the TOPO TA cloning kit (Invitrogen, USA) and transformed into E. coli TOP10 cells. Positive clones were sequenced, and aptamer sequences were analyzed using the Clustal and Mfold structure prediction algorithms.

\section{Secondary structure prediction}

To predict aptamer structural folding, we used the mfold algorithm (http://unafold.rna.albany.edu/? $q=m f o l d / D N A-F o l d i n g-F o r m)$. The modeling was done assuming ionic conditions of $157 \mathrm{mM} \mathrm{Na}{ }^{+}$at $4^{\circ} \mathrm{C}$.

\section{Microscopy observation of aptamer binding}

To visualize aptamer binding on the surface of viable target cells, FAM-labeled ssDNA aptamers (250 nM) were incubated with the target strains $\left(10^{7}\right.$ cells $)$ for $45 \mathrm{~min}$ at $20^{\circ} \mathrm{C}$, and then cells were centrifuged and 
the supernatant discarded. The cells then went through two rounds of washing and centrifugation, with the supernatants again discarded. The cells were resuspended in $40 \mu \mathrm{L}$ of washing buffer. Imaging of the bacteria was performed with a microscope (ZEISS, Axio Scope.A1) under excitation of $488 \mathrm{~nm}$ and emission in the range of 502 to $554 \mathrm{~nm}$.

\section{Aptamer binding analysis}

Aptamer $K_{\mathrm{d}}$ was determined by measuring the fluorescence intensity of each sample. The FAM-labeled ssDNA aptamers (in increasing concentrations of $12.5,31.25,62.5,125,250$, and $500 \mathrm{nM}$ ) were incubated with the target strains $\left(10^{7}\right.$ cells) for $60 \mathrm{~min}$ at $20^{\circ} \mathrm{C}$. Cells were washed two times to remove unbound ssDNA from cells and resuspended in $100 \mu \mathrm{L}$ of washing buffer. The $K_{\mathrm{d}}$ values for each aptamer were determined by nonlinear regression analysis using Origin 9.5 software.

\section{Abbreviations}

FAM, 6-carboxyfluorescein; SELEX, systematic evolution of ligands by exponential enrichment; ELISA, enzyme linked immunosorbent assay; CFU, colony forming units.

\section{Declarations}

\section{Acknowledgments}

We thank Dr. Zhenhuang Ge at Sun Yat-sen University for technical assistance in culture of $L$. pneumophila. We also thank Guangzhou Saite Testing Co., LTD, Guangzhou, China for technical assistance in detecting the characteristics of aptamers.

\section{Availability of data and materials}

All data generated or analyzed during this study are included in this published article, and are available from the corresponding author (sunyunhaoscope@163.com) on reasonable request.

\section{Competing interests}

The authors declare that they have no competing interests.

\section{Funding}

This work was supported by Funds for the Key Scientific and Technological Innovation Projects of Guangdong Forestry Bureau (grant no. 2020KJCX009 to Yunhao Sun, for the collection of data), the National Natural Science Foundation of China (grant no. 32000086 to Yunhao Sun, for the analysis of the data and writing of the manuscript), the Applied Basic Research Programs of Science and Technology Commission Foundation of Guangdong Province (grant no. 2019A1515110593 to Yunhao Sun, for the analysis of the data and writing of the manuscript), the Research Project of Innovative Institute for Plant 
Health from Zhongkai University of Agriculture and Engineering (grant no. KA21031H103 to Yunhao Sun, for the collection of data), and the Starting Research Fund from Zhongkai University of Agriculture and Engineering (grant no. KA200540844 to Yunhao Sun, for the collection of data).

\section{Authors' contributions}

Lina Xiong, Mingchen Xia and Yunhao Sun conceived and designed the study. Lina Xiong, Qinglin Wang, Zhen Meng, Jie Zhang, Guohui Yu and Zhangyong Dong performed the experiments. Lina Xiong, Yongjun Lu and Yunhao Sun wrote the paper. Guohui Yuand Zhangyong Dong reviewed and edited the manuscript. All authors read and approved the manuscript.

\section{References}

1. Bruno, J.G. (2015) Predicting the Uncertain Future of Aptamer-Based Diagnostics and Therapeutics. Molecules, 20, 6866-6887.

2. Cao, X., Li, S., Chen, L., Ding, H., Xu, H., Huang, Y., Li, J., Liu, N., Cao, W., Zhu, Y., Shen, B., Shao, N. (2009) Combining use of a panel of ssDNA aptamers in the detection of Staphylococcus aureus. Nucleic Acids Res, 37, 4621-4628.

3. Casini, B., Aquino, F., Totaro, M., Miccoli, M., Galli, I., Manfredini, L., Giustarini, C., Costa, A.L., Tuvo, B., Valentini, P., Privitera, G., Baggiani, A. (2017) Application of Hydrogen Peroxide as an Innovative Method of Treatment for Legionella Control in a Hospital Water Network. Pathogens, 6.

4. Chen, F., Zhou, J., Luo, F., Mohammed, A.B., Zhang, X.L. (2007) Aptamer from whole-bacterium SELEX as new therapeutic reagent against virulent Mycobacterium tuberculosis. Biochem Biophys Res Commun, 357, 743-748.

5. Cloutman-Green, E., Barbosa, V.L., Jimenez, D., Wong, D., Dunn, H., Needham, B., Ciric, L., Hartley, J.C. (2019) Controlling Legionella pneumophila in water systems at reduced hot water temperatures with copper and silver ionization. Am J Infect Control.

6. Dua, P., Ren, S., Lee, S.W., Kim, J.K., Shin, H.S., Jeong, O.C., Kim, S., Lee, D.K. (2016) Cell-SELEX Based Identification of an RNA Aptamer for Escherichia coli and Its Use in Various Detection Formats. Mol Cells, 39, 807-813.

7. Duan, N., Ding, X., Wu, S., Xia, Y., Ma, X., Wang, Z., Chen, J. (2013) In vitro selection of a DNA aptamer targeted against Shigella dysenteriae. J Microbiol Methods, 94, 170-174.

8. Duan, N., Wu, S., Chen, X., Huang, Y., Wang, Z. (2012) Selection and identification of a DNA aptamer targeted to Vibrio parahemolyticus. J Agric Food Chem, 60, 4034-4038.

9. Duan, N., Wu, S., Chen, X., Huang, Y., Xia, Y., Ma, X., Wang, Z. (2013) Selection and characterization of aptamers against Salmonella typhimurium using whole-bacterium Systemic Evolution of Ligands by Exponential Enrichment (SELEX). J Agric Food Chem, 61, 3229-3234.

10. Dwivedi, H.P., Smiley, R.D., Jaykus, L.A. (2010) Selection and characterization of DNA aptamers with binding selectivity to Campylobacter jejuni using whole-cell SELEX. App/ Microbiol Biotechnol, 87, 
2323-2334.

11. Dwivedi, H.P., Smiley, R.D., Jaykus, L.A. (2013) Selection of DNA aptamers for capture and detection of Salmonella Typhimurium using a whole-cell SELEX approach in conjunction with cell sorting. App/ Microbiol Biotechnol, 97, 3677-3686.

12. Gopinath, S.C., Tang, T.H., Chen, Y., Citartan, M., Lakshmipriya, T. (2014) Bacterial detection: from microscope to smartphone. Biosens Bioelectron, 60, 332-342.

13. Keefe, A.D., Pai, S., Ellington, A. (2010) Aptamers as therapeutics. Nat Rev Drug Discov, 9, 537-550.

14. Kolovskaya, O.S., Savitskaya, A.G., Zamay, T.N., Reshetneva, I.T., Zamay, G.S., Erkaev, E.N., Wang, X., Wehbe, M., Salmina, A.B., Perianova, O.V., Zubkova, O.A., Spivak, E.A., Mezko, V.S., Glazyrin, Y.E., Titova, N.M., Berezovski, M.V., Zamay, A.S. (2013) Development of bacteriostatic DNA aptamers for salmonella. J Med Chem, 56, 1564-1572.

15. Maita, C., Matushita, M., Okubo, T., Matsuo, J., Miyake, M., Nagai, H., Yamaguchi, H. (2016) Draft Genome Sequences of Legionella pneumophila JR32 and Lp01 Laboratory Strains Domesticated in Japan. Genome Announc, 4.

16. Moon, J., Kim, G., Lee, S., Park, S. (2013) Identification of Salmonella Typhimurium-specific DNA aptamers developed using whole-cell SELEX and FACS analysis. J Microbiol Methods, 95, 162-166.

17. Park, H.C., Baig, I.A., Lee, S.C., Moon, J.Y., Yoon, M.Y. (2014) Development of ssDNA aptamers for the sensitive detection of Salmonella typhimurium and Salmonella enteritidis. Appl Biochem Biotechnol, 174, 793-802.

18. Peng, Z., Ling, M., Ning, Y., Deng, L. (2014) Rapid fluorescent detection of Escherichia coli K88 based on DNA aptamer library as direct and specific reporter combined with immuno-magnetic separation. J Fluoresc, 24, 1159-1168.

19. Rajendhran, J., Gunasekaran, P. (2011) Microbial phylogeny and diversity: small subunit ribosomal RNA sequence analysis and beyond. Microbiol Res, 166, 99-110.

20. Savory, N., Lednor, D., Tsukakoshi, K., Abe, K., Yoshida, W., Ferri, S., Jones, B.V., Ikebukuro, K. (2013) In silico maturation of binding-specificity of DNA aptamers against Proteus mirabilis. Biotechnol Bioeng, 110, 2573-2580.

21. Schmolders, J., Manske, C., Otto, A., Hoffmann, C., Steiner, B., Welin, A., Becher, D., Hilbi, H. (2017) Comparative Proteomics of Purified Pathogen Vacuoles Correlates Intracellular Replication of Legionella pneumophila with the Small GTPase Ras-related protein 1 (Rap1). Mol Cell Proteomics, $16,622-641$.

22. Suh, S.H., Dwivedi, H.P., Choi, S.J., Jaykus, L.A. (2014) Selection and characterization of DNA aptamers specific for Listeria species. Anal Biochem, 459, 39-45.

23. Suh, S.H., Jaykus, L.A. (2013) Nucleic acid aptamers for capture and detection of Listeria spp. J Biotechnol, 167, 454-461.

24. Syed, M.A., Jamil, B. (2018) Aptamers and Aptasensors as Novel Approach for Microbial Detection and Identification: An Appraisal. Curr Drug Targets, 19, 1560-1572. 
25. Sypabekova, M., Bekmurzayeva, A., Wang, R., Li, Y., Nogues, C., Kanayeva, D. (2017) Selection, characterization, and application of DNA aptamers for detection of Mycobacterium tuberculosis secreted protein MPT64. Tuberculosis (Edinb), 104, 70-78.

26. Tang, X., Zheng, J., Yan, Q., Li, Z., Li, Y. (2013) Selection of aptamers against inactive Vibrio alginolyticus and application in a qualitative detection assay. Biotechnol Lett, 35, 909-914.

27. Torres-Chavolla, E., Alocilja, E.C. (2009) Aptasensors for detection of microbial and viral pathogens. Biosens Bioelectron, 24, 3175-3182.

28. Wang, K.Y., Zeng, Y.L., Yang, X.Y., Li, W.B., Lan, X.P. (2011) Utility of aptamer-fluorescence in situ hybridization for rapid detection of Pseudomonas aeruginosa. Eur J Clin Microbiol Infect Dis, 30, 273-278.

29. Woo, P.C., Lau, S.K., Teng, J.L., Tse, H., Yuen, K.Y. (2008) Then and now: use of 16 S rDNA gene sequencing for bacterial identification and discovery of novel bacteria in clinical microbiology laboratories. Clin Microbiol Infect, 14, 908-934.

30. Xiong, L., Yamasaki, S., Chen, H., Shi, L., Mo, Z. (2017) Intracellular Growth and Morphological Characteristics of Legionella pneumophila during Invasion and Proliferation in Different Cells. Biol Pharm Bull, 40, 1035-1042.

31. Xiong, L., Yan, H., Shi, L., Mo, Z. (2016) Antibiotic susceptibility of Legionella strains isolated from public water sources in Macau and Guangzhou. J Water Health, 14, 1041-1046.

32. Xiong, L., Zhao, H., Mo, Z., Shi, L. (2015) Prevalence of 7 virulence genes of Legionella strains isolated from environmental water sources of public facilities and sequence types diversity of $\mathrm{L}$. pneumopila strains in Macau. Biosci Trends, 9, 214-220.

33. Yang, M., Peng, Z., Ning, Y., Chen, Y., Zhou, Q., Deng, L. (2013) Highly specific and cost-efficient detection of Salmonella Paratyphi A combining aptamers with single-walled carbon nanotubes. Sensors (Basel), 13, 6865-6881.

\section{Tables}

Table 1. Oligonucleotides used in the selection and characterization of aptamers with binding affinity to Legionella pneumophila. 


\section{Name}

DNA aptamer library

Forward constant region primer

Reverse constant region primer

FAM-forward constant region primer

P-reverse constant region primer

\section{Oligonucleotides}

5'-GCAATGGTACGGTACTTCC-N45-

CAAAAGTGCACGCTACTTTGCTAA-3'(88-mer)

5'-GCAATGGTACGGTACTTCC-3'

5'-TTAGCAAAGTAGCGTGCACTTTTG-3'

5'-FAM-GCAATGGTACGGTACTTCC-3'

5'-phosphate-TTAGCAAAGTAGCGTGCACTTTTG-3'

Table 2. List of four identified Legionella pneumophila binding ssDNA aptamers.

\begin{tabular}{|lll|}
\hline Aptamer & Sequence of N45 random region $\left(5^{\prime}\right.$ to $\left.3^{\prime}\right)$ & $\% G C$ \\
\hline S11 & GTGCGCTCCGTGGGCAACGACACCTTGTTTGAAGTGTTTAGGAAA & 51 \\
\hline S25 & CAGCCGTAACATCAGACAGCTGTAAAAAGCGGGATGTGGACTAGT & 49 \\
\hline S28 & CGCCTGTGGGTGCACAGCAGCCGCGCAGGCTGAAATACTCTGTGT & 62 \\
\hline S29 & TCGGTAAGGGCAGTCTTAGCGTGGTTCCATCTGTACCGAAGCCGT & 56 \\
\hline
\end{tabular}

\section{Figures}

A

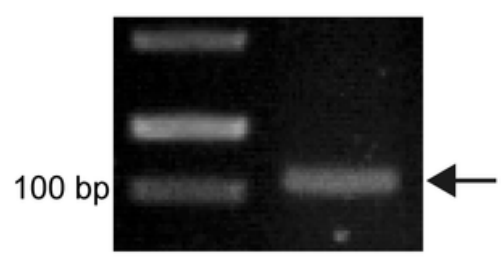

B

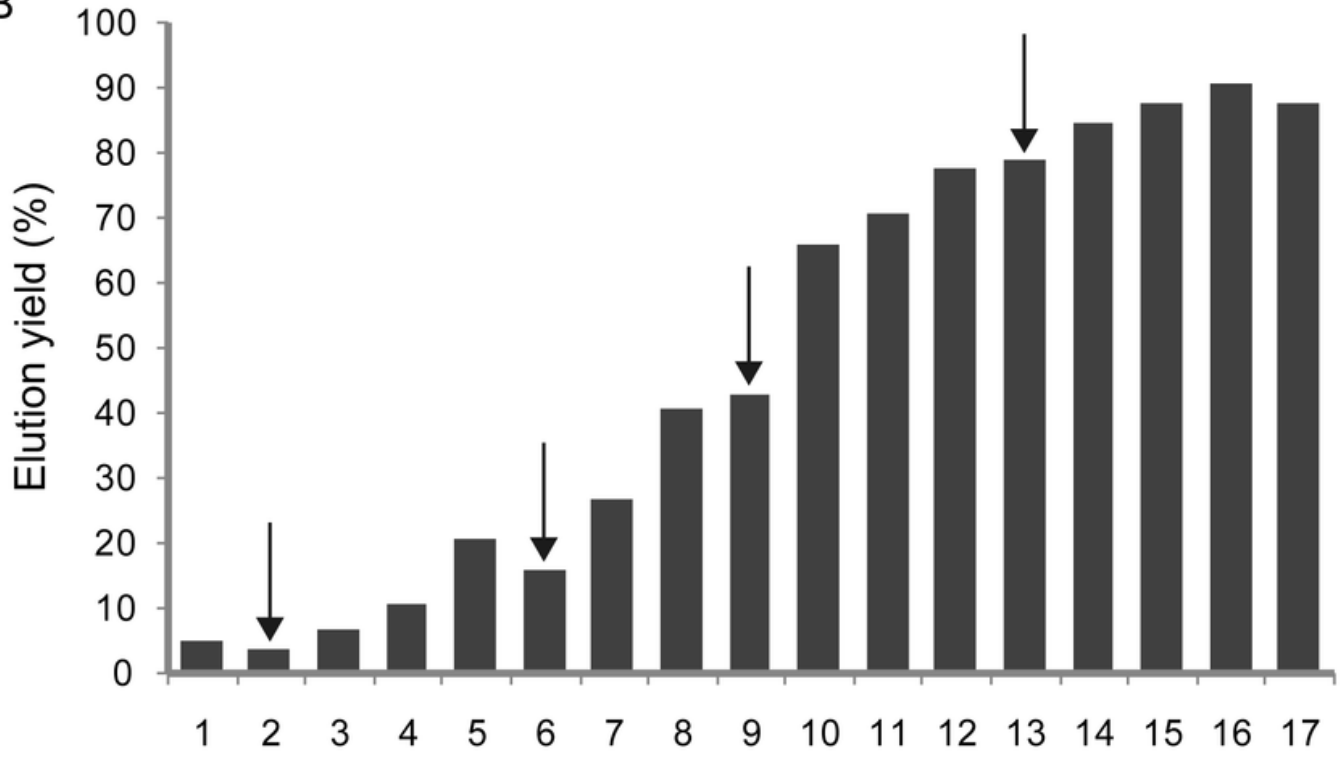


Figure 1

In vitro selection of aptamers. A. The PCR products of the 9th round of selection were analyzed by agarose gel electrophoresis. Lane 1, DNA ladder; lane 2, amplified DNA products. Black arrow indicates the PCR product band. B. Progression of the selection process based on the portion of ssDNA bound to the target cells in the DNA pool. Numbers 1-17 represent the seventeen rounds of selection. Black arrows indicate the rounds of counter selection.
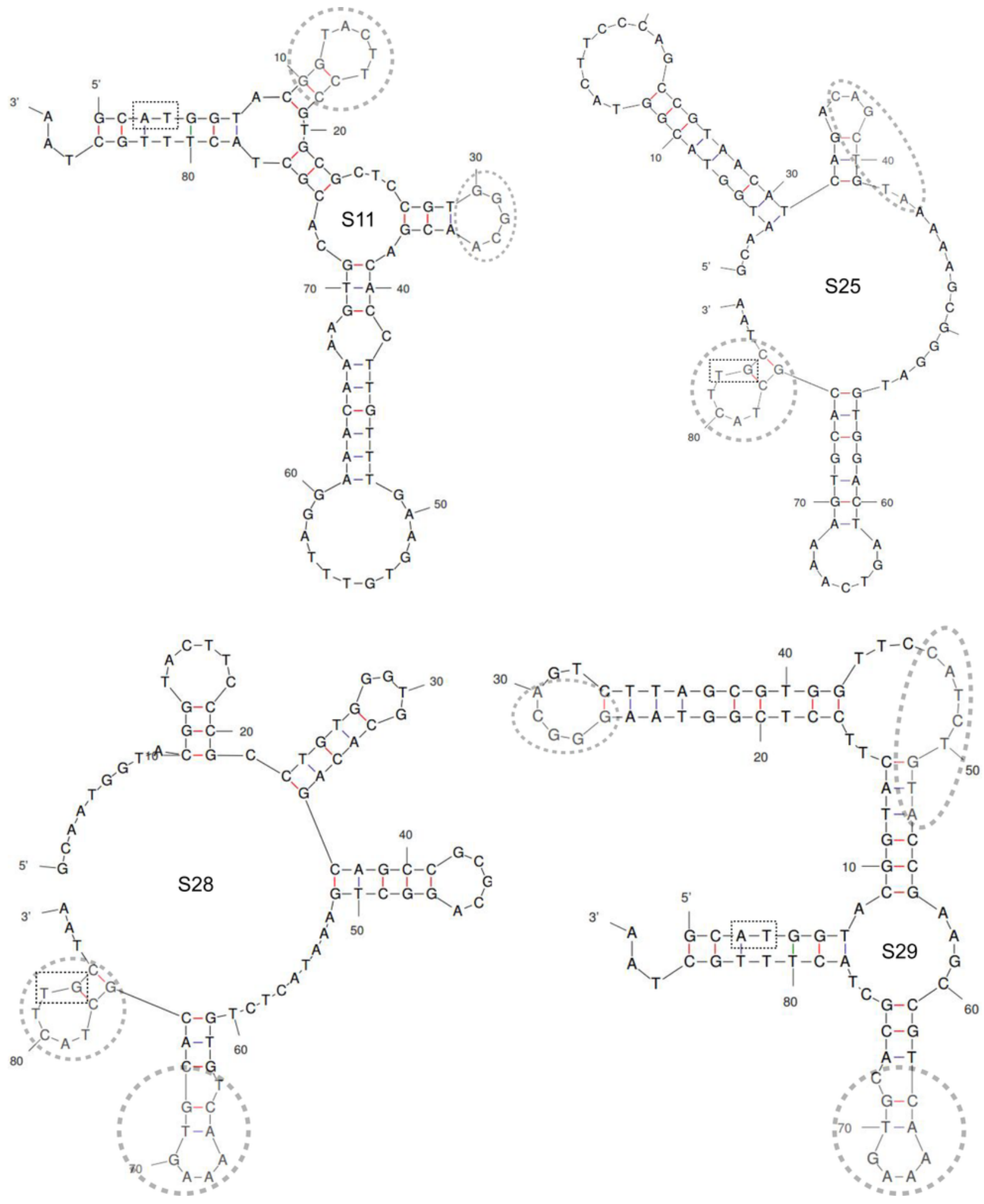
Figure 2

Predicted secondary structures of four obtained aptamers (S11, S25, S28, and S29). The DNA folding platform from the mfold web server based on a free energy minimization algorithm was used. The conserved stem loops are depicted in dotted circles.

A

JR32
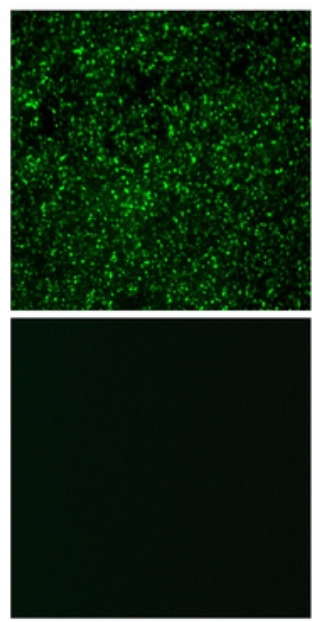

Escherichia coli

Pseudomonas syringae
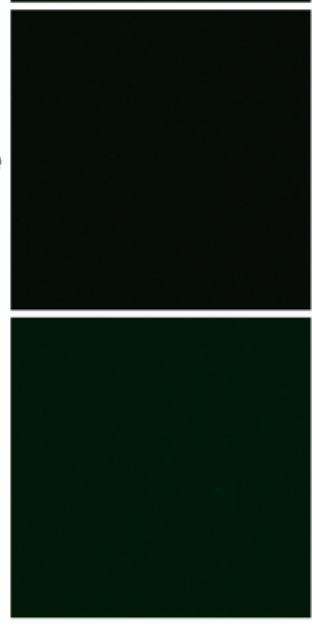

Legionella quateirensis

B

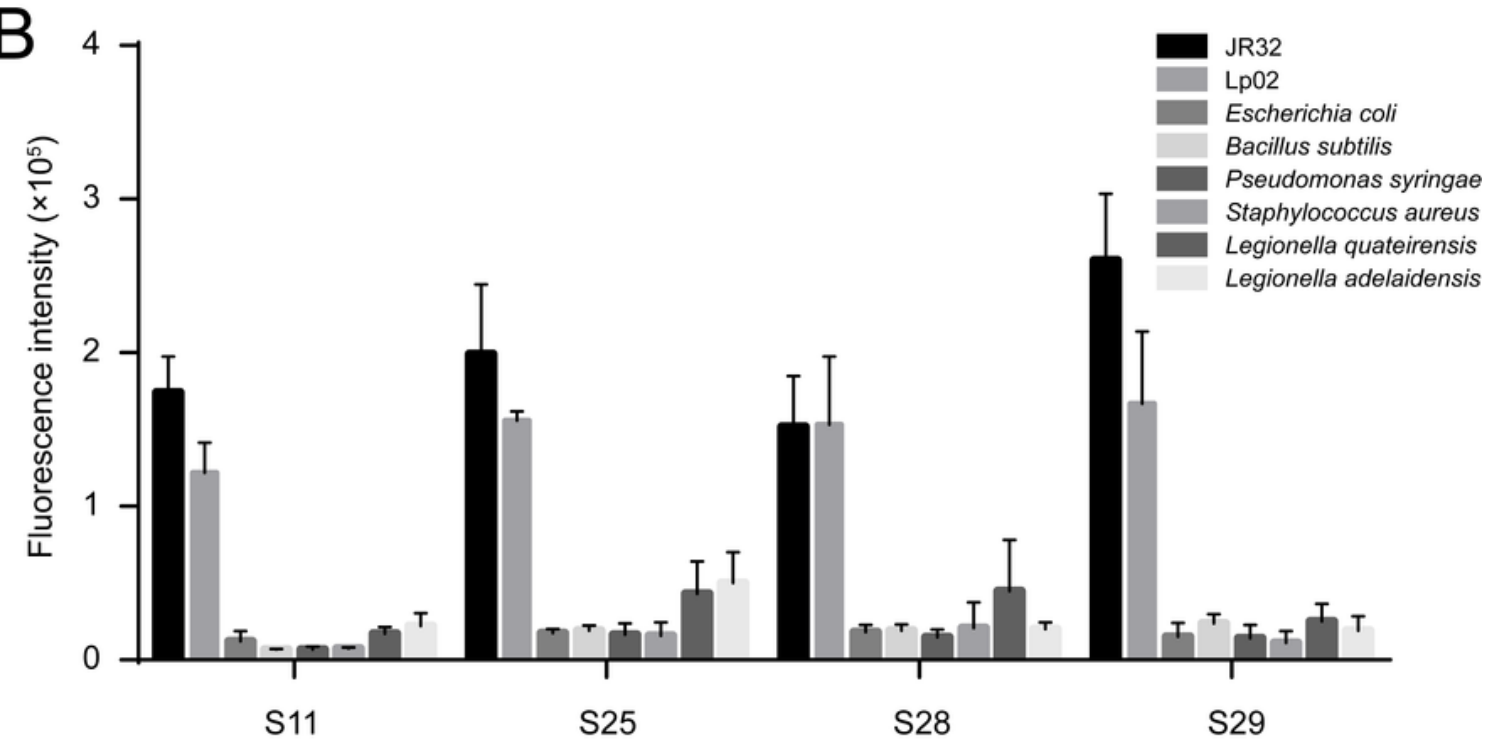

Bacillus subtilis

Staphylococcus aureus

Legionella adelaidensis
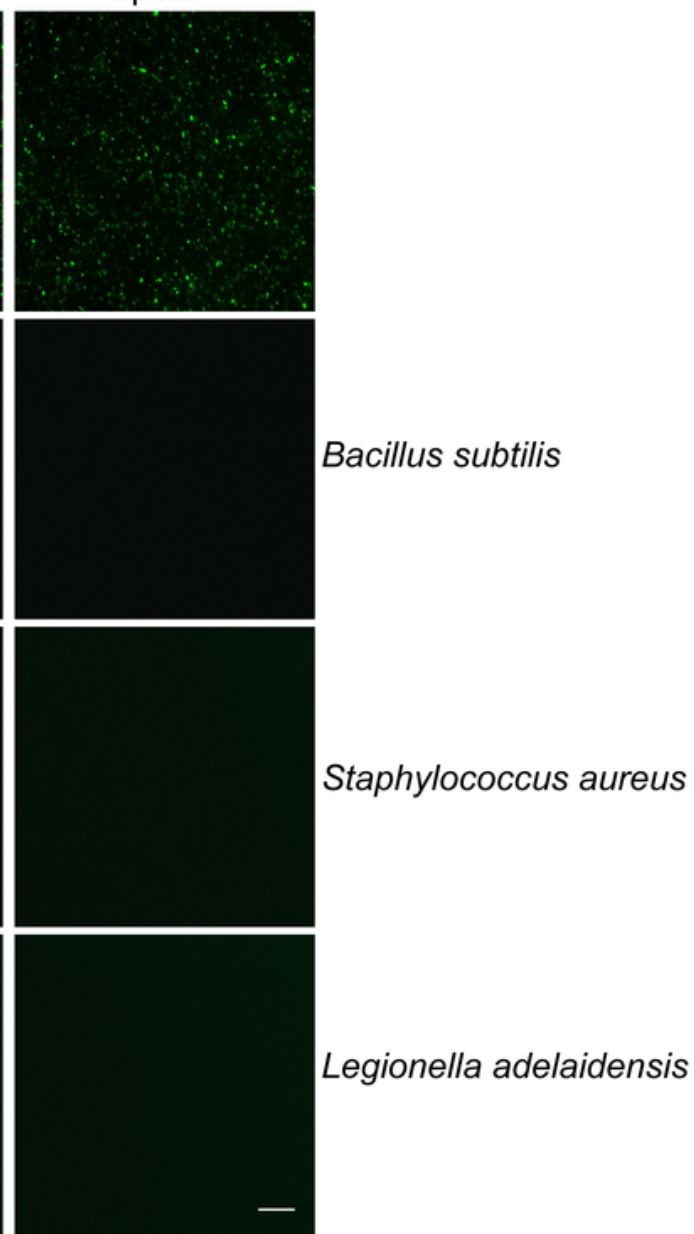

Figure 3 
Detection and specificity of aptamers for Legionella pneumophila. A. Fluorescein-labeled ssDNA aptamers (250 nM in binding buffer; representative pictures of aptamer S29 are shown) were incubated with $10^{7}$ bacteria at $20^{\circ} \mathrm{C}$ for $45 \mathrm{~min}$ (scale bar, $10 \mu \mathrm{m}$ ). B. Selectivity of aptamers (S11, S25, S28, and S29) for Legionella pneumophila, Escherichia coli, Bacillus subtilis, Pseudomonas syringae, Staphylococcus aureus, Legionella quateirensis, and Legionella adelaidensis cells.
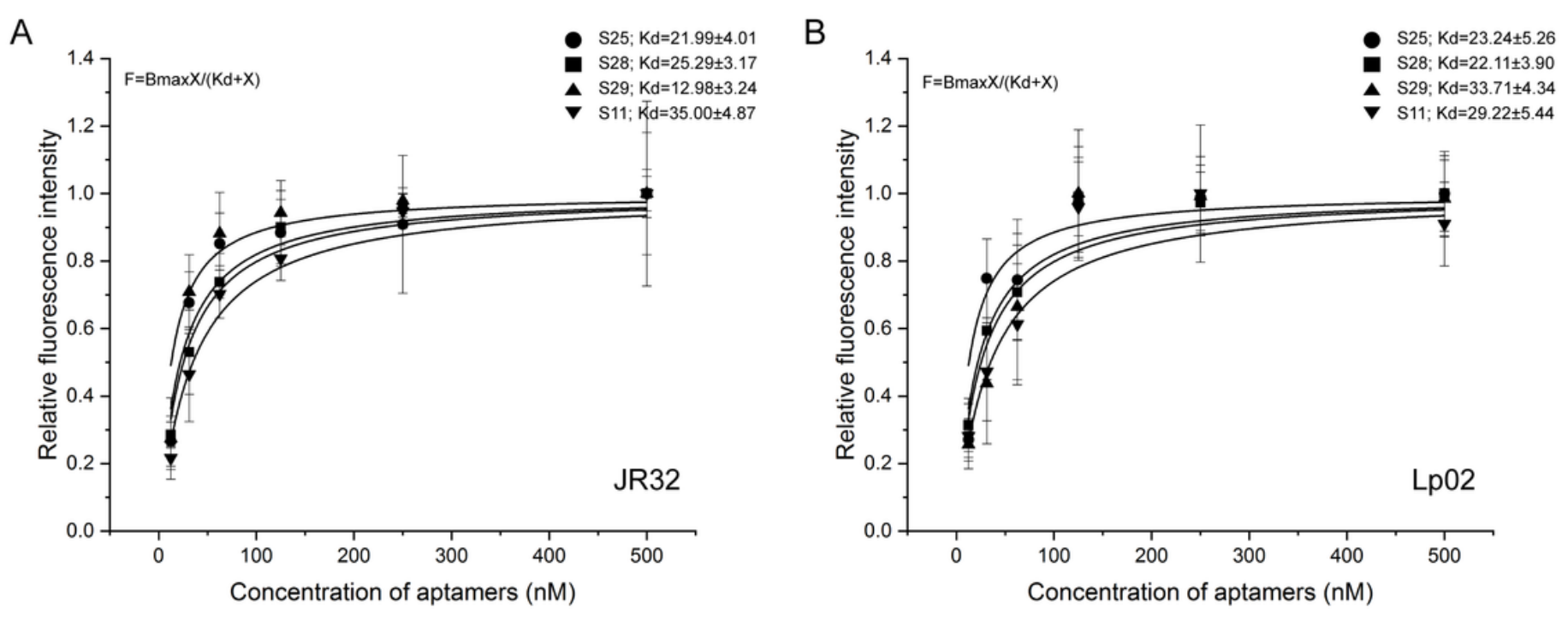

\section{Figure 4}

Binding affınity of four selected aptamers for Legionella pneumophila.

The 6-carboxyfluorescein-labeled ssDNA aptamers (in increasing concentrations of $12.5,31.25,62.5$,

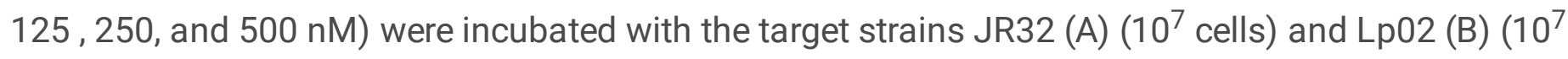
cells) for $60 \mathrm{~min}$ at $20^{\circ} \mathrm{C}$. The $K_{\mathrm{d}}$ values for each aptamer were determined by nonlinear regression analysis using Origin 9.5 software. Data represent the mean SD of three independent experiments. 